\title{
Anisotropic diffusion of tagged spheres near the isotropic-nematic phase transition
}

\author{
R. Vasanthi \\ Solid State and Structural Chemistry Unit, Indian Institute of Science, Bangalore, India 560012 \\ S. Ravichandran \\ University of Medicine and Dentistry of New Jersey, Newark, New Jersey 07103 \\ Biman Bagchia) \\ Solid State and Structural Chemistry Unit, Indian Institute of Science, Bangalore, India 560012
}

\begin{abstract}
Molecular dynamics simulations of diffusion of isolated tagged spheres in liquid crystalline molecules have been carried out. While the diffusion is isotropic at density slightly below the isotropic-nematic phase transition, it is found to become strongly anisotropic very close to the I-N transition where the diffusion perpendicular to the direction of the order parameter $g\left(D_{\perp}\right)$ decouples from the parallel $\left(D_{\|}\right)$component. The diffusion coefficient of the sphere also shows a marked slowing down as the I-N transition approaches, in accordance with the mode coupling theory analysis. The variation of the orientational order parameter with the density shows a sharp transition at a reduced density, $\rho_{\text {IN }}^{*}$ where plot of the ratio, $D_{\|} / D_{\perp}$ against the reduced density, $\rho^{*}$ clearly shows the emergence of anisotropy. $D_{\perp}$ could be fitted to a form $\left(\rho_{c}^{*}-\rho^{*}\right)^{p}$ with a $\rho_{c}^{*}$ which is slightly larger than $\rho_{\mathrm{IN}}^{*}$. The diffusion shows a strong non-Stokesian dependence on the radius of the spheres, in agreement with recent experiments.
\end{abstract}

\section{INTRODUCTION}

The dynamics of the anisotropic molecules ${ }^{1}$ are considerably more complicated than those of the spherical molecules because orientational correlations can have profound effects on both translational and rotational motion of the molecules. The simplest way to characterize the anisotropy of the molecule is to model it as an ellipsoid. The anisotropy in the molecular shape is then characterized by the aspect ratio $\kappa$, which is the ratio of the length of the major axis (a) to that of the minor axis (b), i.e., $\kappa=a / b$. When $\kappa \gg 1$, i.e., when the molecule is rodlike, the system can undergo a phase transition from nematic to the isotropic phase. This phase transition is weakly of first order with many characteristics similar to those observed near the critical point. The orientational order parameter $S$ of this transition can be defined by ${ }^{2-5}$

$$
S=\frac{1}{2 N}\left\langle\sum_{i=1}^{N}\left(3 \cos ^{2} \theta_{i}-1\right)\right\rangle,
$$

where $\theta_{i}$ is the angle of the ellipsoidal axis with the Z-axis of an arbitrary space fixed frame. In the isotropic phase, the value of the order parameter $S$ is zero, whereas in the nematic phase, where the ellipsoids are oriented nearly parallel to each other, the order parameter is unity. Near the isotropicnematic (I-N) phase transition, the order parameter $S$ shows a dynamic slowing down in the sense that the decay of the time correlation function, $\langle S(0) S(t)\rangle$ becomes very slow.

\footnotetext{
a) Author to whom correspondence should be addressed; electronic mail: bbagchi@sscu.iisc.ernet.in
}

Here $S(t)$ is the instantaneous order parameter. This slowing down has been attributed to the formation of correlated domains, where the ellipsoidal or rodlike molecules are nearly parallel. The understanding of the collective dynamics near the I-N transition has been a subject of interest for a long time. $^{4}$

In recent years, several computer simulation studies have been carried out in order to obtain an understanding of the dynamics at the microscopic level. These simulations employed either a hard repulsive interaction potential ${ }^{6}$ or the Lennard-Jones-like Gay-Berne potential and have been successful $^{7-11}$ in producing I-N phase transition with the characteristics observed by the experiments, although the aspect ratio of the ellipsoids or the rods simulated were much smaller than those usually found in the real world. For example, one finds a good I-N transition for the prolates with aspect ratio, $\kappa=3$. In Fig. 1, we show the simulated order parameter variation with density. The transition occurs sharply at $\rho^{*}=0.315$. The simulation studies have also found that a power-law-like behavior in orientational relaxation occurs near the I-N transition. ${ }^{8}$ Recent experimental studies by Fayer and co-workers ${ }^{12}$ have revealed many fascinating aspects of short and intermediate time of the dynamics near the I-N transition. This includes the observation of a power law decay at intermediate times. ${ }^{13}$ Generalized hydrodynamic equations have been used to understand these results with some degree of success, especially in exploring long time orientational dynamics in the isotropic phase. No fully satisfactory explanation of the intermediate time power law decay has yet been put forward, although work in this direction is under progress. ${ }^{14}$ 
Recently experiments have been performed to study the dynamics of tagged spheres in rigid colloidal hard rods. ${ }^{15}$ It has been observed that in the limit of large volume fractions of the hard rods, the diffusion of the tagged spheres depends strongly on the size of the spheres. ${ }^{15}$ We also find a similar result. The diffusion coefficient of the sphere show a nonStokesian dependence on the radius of the sphere. In this work, molecular dynamics computer simulation of diffusion of a few isolated tagged spheres in a liquid of prolate ellipsoids with aspect ratio, $\kappa=3$, has been presented. This system undergoes an I-N phase transition at a reduced density $\rho^{*}=0.315$ with $T^{*}=1.0$, as shown in Fig. 1 . The reason for studying the diffusion of tagged spherical molecules in this system is to explore the possibility of using this method to study the dynamics of nematogens near the I-N transition. ${ }^{4}$ We have varied the size of the tagged spheres which has revealed several interesting aspects. As the I-N transition is approached, we find that the total diffusion coefficient of all the spheres of different sizes decreases, but rather differently. We also find that the diffusion coefficient becomes anisotropic very close to the I-N transition region and is also dependent on the size of the spheres.

The rest of the paper is organized as follows. In the next section, we discuss the mode coupling theory approach, followed by details about the systems studied, the interaction potential used and also the simulation procedure. In Sec. IV, we present the results and in Sec. V, we conclude with a brief discussion.

\section{MODE COUPLING THEORY OF TAGGED PARTICLE DIFFUSION NEAR THE ISOTROPIC-NEMATIC PHASE TRANSITION}

Near the I-N transition, the collective orientational relaxation slows down dramatically. This should certainly affect the translational diffusion of the tagged spheres. However, we are not aware of any theoretical study of this translationrotation coupling. Here we present a theoretical study of this effect by using the mode coupling theory. ${ }^{16,17}$ We start with Einstein's relation between diffusion $(D)$ and friction coefficient $(\zeta)$ on the sphere

$$
D=\frac{k_{B} T}{\zeta} \text {. }
$$

We split friction $\zeta$ into a short time (binary) and a collective term

$$
\zeta=\zeta_{\text {bin }}+\zeta_{\rho \rho},
$$

where we have approximated the collective contribution by the density mode. This should be okay as we treat the effects of slow orientational density relaxation. $\zeta_{\rho \rho}$ can be obtained from Kirkwood's formula

$$
\zeta_{\rho \rho}=\frac{1}{3 k_{B} T V} \int_{V} d \mathbf{r} \int_{0}^{\infty} d t\langle\mathbf{F}(\mathbf{r}, 0) . \mathbf{F}(\mathbf{r}, t)\rangle,
$$

where $V$ is the total volume of the system. The fluctuation force $\mathbf{F}(t)$ is now obtained from the density functional theory $^{18}$

$$
\beta \mathbf{F}(\mathbf{r}, t)=\nabla \int d \mathbf{r}^{\prime} \int d \mathbf{\Omega}^{\prime} \mathbf{c}\left(\mathbf{r}-\mathbf{r}^{\prime}, \boldsymbol{\Omega}^{\prime}\right) \delta \rho_{\mathbf{N}}\left(\mathbf{r}^{\prime}, \mathbf{\Omega}^{\prime}, \mathbf{t}\right),
$$

where $\delta \rho_{N}\left(\mathbf{r}^{\prime}, \mathbf{\Omega}^{\prime}, t\right)$ is the fluctuation in the density of the nematogens,

$$
\delta \rho_{N}=\rho_{N}-\bar{\rho} / 4 \pi,
$$

where $\bar{\rho}$ is the average number density of the nematogens, $c\left(\mathbf{r}-\mathbf{r}^{\prime}, \mathbf{\Omega}^{\prime}\right)$ is the two particle direct correlation function between the tagged sphere at position $\mathbf{r}$ and the nematogen at $\mathbf{r}^{\prime}$ with the orientation $\boldsymbol{\Omega}^{\prime}$. We now split $\zeta_{\rho \rho}$ further into two terms:

$$
\zeta_{\rho \rho}=\zeta_{\rho \rho}^{N}+\zeta_{\rho \rho}^{\operatorname{sing}},
$$

where $\zeta_{\rho \rho}^{N}$ is the normal part due to isotropic interaction between the sphere and the nematogens (approximated here as ellipsoids). $\zeta_{\rho \rho}^{\text {sing }}$ is the singular part due to I-N transition. $\zeta_{\rho \rho}^{\text {sing }}$ derives maximum contribution from coupling to the orientational order parameter, that is, to the $Y_{20}(\Omega(t))$ component of the nematogen density. In the subsequent steps we transform the equations to the wave number $k$ space, expand both the direct correlation function and the density in spherical harmonics, carry out the angular integrations to obtain

$$
\zeta_{\rho \rho}^{\operatorname{sing}}=A \int d k k^{4} c_{20}^{2}(k) F_{20}(k, t),
$$

where $c_{20}$ is the (20) component of the wave number dependent direct correlation function between the sphere and the nematogen and $A$ is a numerical constant. $F_{20}(k, t)$ is the orientational intermediate scattering function given by

$$
F_{20}(k, t)=\left\langle\delta \rho_{20}(-\mathbf{k}, t=0) \delta \rho_{20}(\mathbf{k}, t)\right\rangle,
$$

with

$$
\delta \rho(\mathbf{k}, \mathbf{\Omega}, t)=\sum \delta \rho_{l m}(\mathbf{k}, t) Y_{l m}(\Omega(t)) .
$$

The decay of $F_{20}(k, t)$ becomes very slow at small wave numbers near the I-N transition. $F_{20}(k, t)$ can be approximated by

$$
F_{20}(k, t)=S_{220}(k) \exp \left(-\frac{6 D_{R} t}{S_{220}(k)}\right),
$$

where

$$
S_{220}(k)=F_{20}(k, t=0) \equiv\left\langle\delta \rho_{20}(-\mathbf{k}) \delta \rho_{20}(\mathbf{k})\right\rangle .
$$

Thus, the $S_{220}(k)$ is the orientational structure factor of the nematogens. Near the I-N transition, $S_{220}(k)$ shows a rapid growth at small $k$ which can be represented as ${ }^{19}$

$$
S_{220}(k) \simeq \frac{k_{0}^{2}}{B k_{0}^{2}+k^{2}},
$$

where

$$
k_{0}^{2}=\left[\left(\frac{\rho}{4 \pi}\right) \frac{\partial^{2}}{\partial k^{2}} c_{220}(k=0)\right],
$$

and 


$$
B=1-\frac{\rho}{4 \pi} c_{220}(k=0) .
$$

We assume that $B$ goes to zero as $\left(\rho_{c}-\rho\right)^{p}$. Therefore, near the transition,

$$
S_{220} \simeq \frac{k_{0}^{2}}{k^{2}}
$$

Use of Eq. (16) in Eq. (8) shows that any singular contribution to $\zeta_{\rho \rho}^{\text {sing }}$ must come from the rotational diffusion coefficient $D_{R}$ of the nematogens. $D_{R}$ is related to the rotational friction $\zeta_{R}$ by

$$
D_{R}=\frac{k_{B} T}{\zeta_{R}} .
$$

A similar mode coupling calculation is now performed to obtain the following contribution to the singular part of rotational friction:

$$
\begin{aligned}
\zeta_{R}^{\operatorname{sing}} & \propto \int_{0}^{\infty} d t \int_{0}^{\infty} d k k^{2} c_{220}^{2} \exp \left(-\frac{D_{R} t}{S_{220}(k)}\right), \\
& \propto \int_{0}^{\infty} d k k^{2} c_{220}^{2}(k) S_{220}(k) / D_{R} .
\end{aligned}
$$

Since $D_{R}$ depends on $\zeta_{R}^{\text {sing }}$, a self-consistent calculation is required. Here we just carry out a one loop calculation to obtain

$$
\zeta_{R}^{\operatorname{sing}} \propto \int_{0}^{\infty} d k \frac{k^{2} k_{0}^{2}}{B k_{0}^{2}+k^{2}} .
$$

$\zeta_{R}^{0}$ is the bare friction. Near the $\mathrm{I}-\mathrm{N}$ transition, $B \simeq 0$, so the singular contribution is non-negligible (unlike the translational friction). We rewrite the above equation as

$$
\zeta_{R}^{\operatorname{sing}} \propto \frac{\zeta_{R}^{0}}{B} \int_{0}^{\infty} d k \frac{\left(k^{2} / B\right) k_{0}^{2}}{k_{0}^{2}+\left(k^{2} / B\right)} .
$$

If $B$ goes to zero as $\left(\rho_{c}-\rho\right)^{p}$, then the first loop calculation predicts a power law slow down of $D_{R}$. This in turn predicts that near the I-N transition, the translation diffusion of a tagged sphere may have a power law dependence of the following type:

$$
D_{T} \propto\left(\rho_{c}-\rho\right)^{p}
$$

where $\rho_{c}$ is a critical point where $S_{220}(k)$ diverges. This point should lie beyond the I-N thermodynamic transition point, $\rho_{\text {IN }} \cdot{ }^{19,20}$ Note that the above analysis is only qualitative and the normal contributions can mask the singular contribution. The main merit of the above analysis is that is shows the slow down of translational diffusion due to slow down in rotational diffusion as the I-N transition is approached. This is indeed a good example of translationrotation coupling, often discussed in literature. Another point of interest is the size dependence of the tagged particle diffusion. This enters through the sphere-nematogen coupling constant $c_{20}(k)$ of Eq. (8).

\section{THE SYSEM AND THE SIMULATION DETAILS}

We have carried out molecular dynamics simulations of 576 molecules consisting of 572 ellipsoids and 4 spheres with constant number $(N)$, volume $(V)$, and energy $(E)$ being the constraints. The ellipsoids are characterized by the aspect ratio, $\kappa$ which is the ratio of the semimajor axis (a) to the semiminor axis (b). ${ }^{9}$

The system is modeled by three different kinds of interactions. The ellipsoid-ellipsoid interaction is given by the Gay-Berne potential, ${ }^{6,7}$ in which the orientation of the ellipsoid is also taken into account. The interaction is given by

$$
\begin{aligned}
U_{\mathrm{GB}}= & 4 \epsilon\left(\hat{r}, \hat{u}_{1}, \hat{u}_{2}\right)\left[\left[\frac{\sigma_{0}^{\prime}}{r-\sigma\left(\hat{r}, \hat{u}_{1}, \hat{u}_{2}\right)+\sigma_{0}^{\prime}}\right]^{12}\right. \\
& \left.-\left[\frac{\sigma_{0}^{\prime}}{r-\sigma\left(\hat{r}, \hat{u}_{1}, \hat{u}_{2}\right)+\sigma_{0}^{\prime}}\right]^{6}\right],
\end{aligned}
$$

with

$$
\begin{aligned}
\sigma\left(\hat{u}_{1}, \hat{u}_{2}, \hat{r}\right)= & \sigma_{0}^{\prime}\left[1-\frac{1}{2} \chi\left[\frac{\left(\hat{r} \cdot \hat{u}_{1}+\hat{r} \cdot \hat{u}_{2}\right)^{2}}{1+\chi\left(\hat{u}_{1} \cdot \hat{u}_{2}\right)}\right.\right. \\
& \left.\left.+\frac{\left(\hat{r} \cdot \hat{u}_{1}-\hat{r} \cdot \hat{u}_{2}\right)^{2}}{1-\chi\left(\hat{u}_{1} \cdot \hat{u}_{2}\right)}\right]\right]^{-1 / 2}
\end{aligned}
$$

where

$$
\sigma_{0}^{\prime}=2 b,
$$

$b$ is the length of the semi-minor axis of the ellipsoid and

$$
\chi=\frac{\sigma_{\|}^{2}-\sigma_{\perp}^{2}}{\sigma_{\|}^{2}+\sigma_{\perp}^{2}} .
$$

The orientation dependent strength parameter is

$$
\begin{aligned}
\epsilon\left(\hat{r}, \hat{u}_{1}, \hat{u}_{2}\right)= & \epsilon_{0}\left[1-\chi^{2}\left(\hat{u}_{1} \cdot \hat{u}_{2}\right)\right]^{-1 / 2} \\
& \times\left[1-\frac{\chi^{\prime}}{2}\left[\frac{\left(\hat{r} \cdot \hat{u}_{1}+\hat{r} \cdot \hat{u}_{2}\right)^{2}}{1+\chi^{\prime} \hat{u}_{1} \cdot \hat{u}_{2}}+\frac{\left(\hat{r} \cdot \hat{u}_{1}-\hat{r} \cdot \hat{u}_{2}\right)^{2}}{1-\chi^{\prime} \hat{u}_{1} \cdot \hat{u}_{2}}\right]\right]^{2},
\end{aligned}
$$

$\chi^{\prime}$ is used to adjust the ratio of side-by-side to end-to-end well depths. $\kappa^{\prime}$ is the ratio of the potential well depths for the side-by-side and end-to-end configurations. In our simulation, the aspect ratio, $\kappa=3$ with $\kappa^{\prime}=5$, and hence, $\chi^{\prime}=0.382$ which is related to $\kappa^{\prime}$ as

$$
\chi^{\prime}=\frac{\sqrt{\kappa^{\prime}}-1}{\sqrt{\kappa^{\prime}}+1} .
$$

The interaction between the spheres is given by the Lennard-Jones potential

$$
U_{\mathrm{LJ}}=4 \epsilon_{0}\left[\left[\frac{\sigma_{0}}{r}\right]^{12}-\left[\frac{\sigma_{0}}{r}\right]^{6}\right],
$$

where $\sigma_{0}$ is the diameter of the sphere, $r$ is the interparticle distance, and $\epsilon_{0}$ is the strength parameter.

The interaction between the spheres and ellipsoids is modeled by the GB-LJ potential ${ }^{21}$ which is given as 


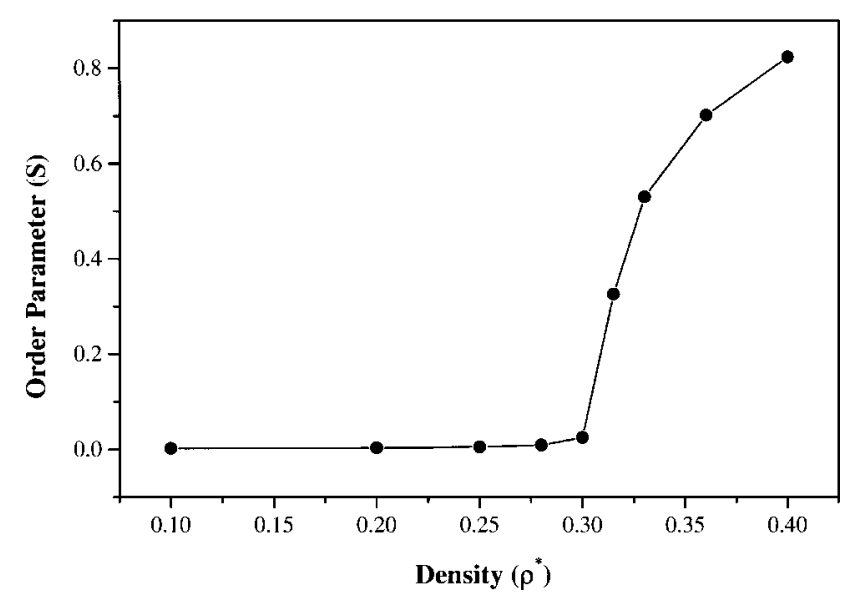

FIG. 1. The variation of the orientational order parameter with the reduced density, $\rho^{*}$ at a reduced temperature, $T^{*}=1.0$. The filled circles are the simulated points. The solid line is for guidance to the eye.

$$
U_{\mathrm{GB}-\mathrm{LJ}}=4 \epsilon_{0}\left[\left[\frac{\sigma(\theta)}{r}\right]^{12}-\left[\frac{\sigma(\theta)}{r}\right]^{6}\right],
$$

with

$$
\sigma(\theta)=\frac{b+R}{\sqrt{1-X^{2} \cos ^{2}(\theta)}},
$$

where $\theta$ is the angle between the major axis of the ellipsoid and the vector joining the centers of the spheres and the ellipsoid and $b$ is the length of the semi-minor axis of the ellipsoid

$$
R=\frac{\sigma_{0}}{2}
$$

and

$$
X=\sqrt{\frac{\bar{a}^{2}-b^{2}}{\bar{a}^{2}+R^{2}}}
$$

with

$$
\bar{a}=\sqrt{\left(b^{2}+R^{2}\right)\left(\frac{a+R}{b+R}\right)^{2}-R^{2}} .
$$

The potential has been cut and shifted to half the boxlength. The translational and the rotational equations of motions are solved by the leap frog algorithm ${ }^{22}$ with reduced moment of inertia being unity $I^{*}=I / m \sigma_{0}^{\prime 2}$. All quantities are scaled. The reduced density is $\rho^{*}=\rho \sigma_{0}^{\prime 3}$ and temperature $T^{*}=k_{B} T / \epsilon_{0}$. All the calculations have been carried out with a time step $\Delta t=0.002$ and at a reduced temperature $T^{*}$ $=1.0$. The simulations have been carried out for three different sphere radii, $0.5,1.0,1.5$, and several densities ranging from 0.1 to 0.4 .

The orientational order parameter $S$ has been calculated by diagonalizing $Q,{ }^{23}$ which is a traceless, symmetric second rank tensor with three eigenvalues and is given by

$$
Q_{\alpha \beta}=\frac{1}{N} \sum_{j=1}^{N} u_{j \alpha} u_{j \beta}-\frac{\delta_{\alpha \beta}}{3},
$$

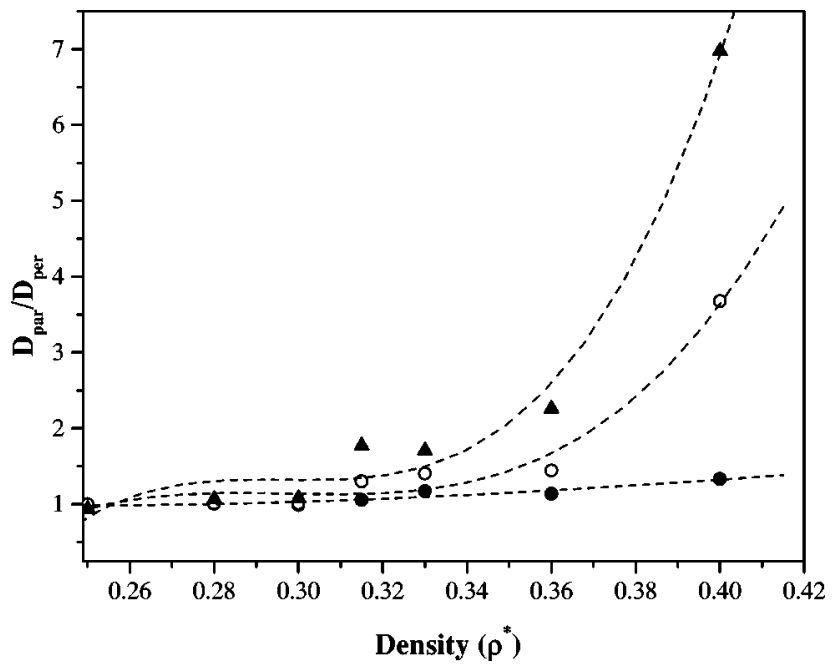

FIG. 2. The plot of the ratio, $D_{\|} / D_{\perp}$, against the reduced density, $\rho^{*}$ at a reduced temperature, $T^{*}=1.0$. The filled circles, open circles, and filled triangles represent the simulated points for spheres of radii $0.5,1.0,1.5$, respectively. The dashed line gives the corresponding fitting to the simulated points.

where $u_{j}$ is the unit vector along the axis of the $j$ th molecule, $u_{j \alpha}$ and $u_{j \beta}$ being the Cartesian coordinates in an orthogonal frame, $\alpha$ and $\beta$ vary between 1 and 3 . The order parameter is then given by $\frac{3}{2}\langle\lambda\rangle$ where $\lambda$ is the largest eigenvalue of the Q-matrix.

The order parameter ${ }^{23}$ can also be calculated by finding the trace of the $Q^{2}$ matrix as given below:

$$
S=\frac{3}{2}\left\langle\operatorname{Tr} Q^{2}\right\rangle \text {. }
$$

Both lead to the same value of $S$.

The anisotropic diffusion coefficients $D_{\|}$and $D_{\perp}$ are calculated by finding the parallel and perpendicular components of mean square displacements. The parallel and the perpendicular components of MSD are parallel and perpendicular to the instantaneous orientational direction of the ellipsoids, respectively. The unit vectors along the direction of average orientation of ellipsoids are the eigenvectors corresponding to the maximum eigenvalue obtained by diagonalizing the time dependent Q-matrix. ${ }^{5}$ Note that there is no ambiguity in finding the eigenvectors for the system with densities above 0.3 in a very long time limit.

\section{RESULTS AND DISCUSSION}

In Fig. 1, we show the variation of the orientational order parameter with the reduced density, $\rho^{*}$. We see that, the order parameter $S$ remains nearly zero until $\rho^{*}=0.3$ and there is a sharp rise in the order parameter at $\rho^{*}=0.315$ and hence the transition is observed at $\rho_{\mathrm{IN}}^{*}=0.315$. As the density is further increased, the order parameter gradually increases toward unity. The time correlation function of the order parameter shows a dramatic slowing down as the I-N transition is approached from the isotropic side. ${ }^{8}$

The diffusion is expected to become anisotropic as the nematic domains appear in the system. However, the local order parameter of these domains varies. But, the direction of these domains does not vary much on average over a long 

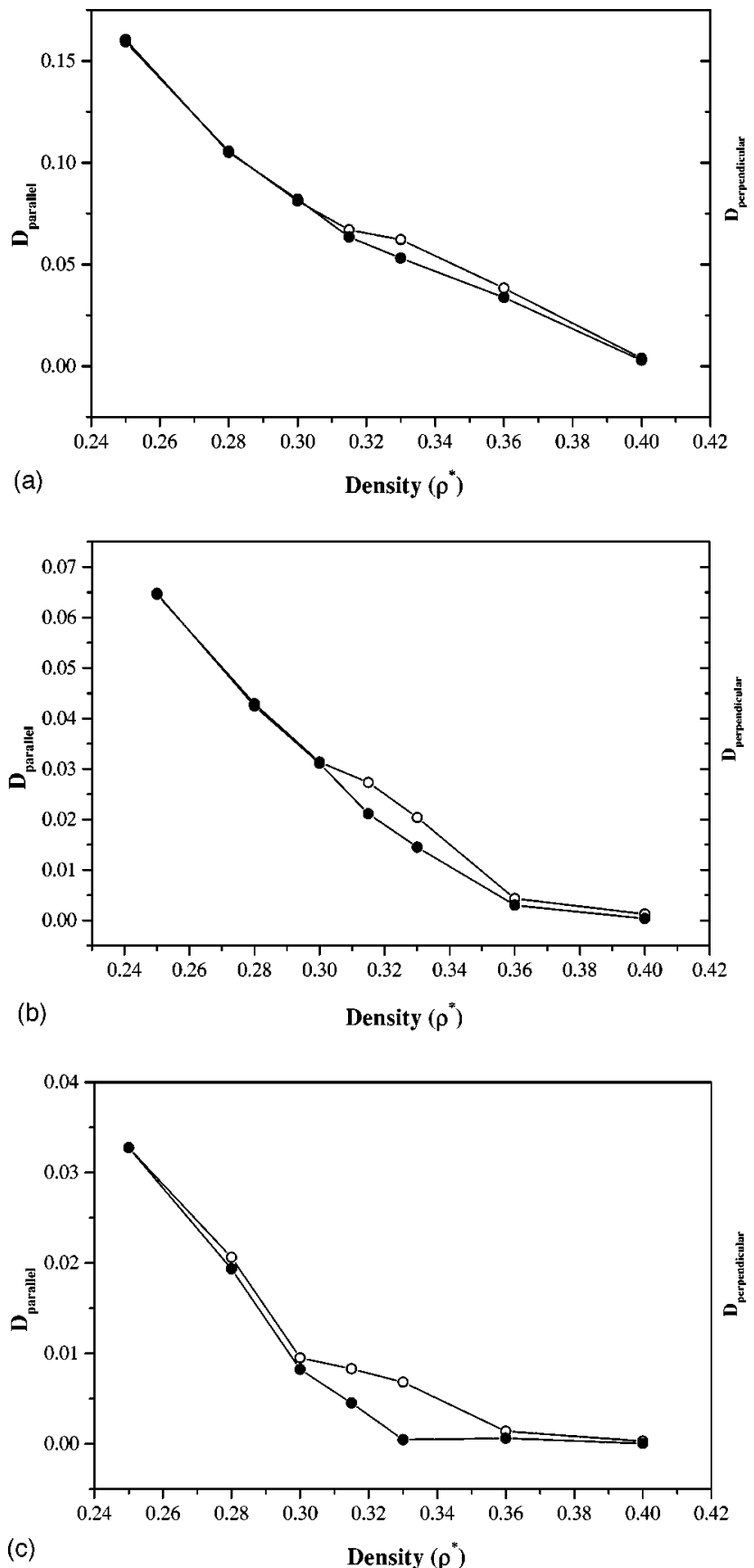

FIG. 3. The variation of the parallel, $D_{\|}$and the perpendicular, $D_{\perp}$ components of diffusion coefficient with the reduced density, $\rho^{*}$ obtained from the simulations at a reduced temperature, $T^{*}=1.0$ for the spheres of different sizes. (a) shows the result for spheres with radius $0.5 \sigma$, (b) represents that for $1.0 \sigma$, and (c) for $1.5 \sigma$. In all the figures, filled and open circles represent $D_{\|}$and $D_{\perp}$, respectively. The error bars are within the radius of the circle.

time. $D_{\|}$and $D_{\perp}$ are defined parallel and perpendicular with respect to the direction of alignment of these domains on an average (globally). The direction of these domains is given by the direction corresponding to the order parameter. In Fig. 2, we plot the ratio $D_{\|} / D_{\perp}$ against density. It is observed that the ratio remains near unity up to a certain density, $\rho^{*}=0.3$ and then increases differently for different sizes. We also observe that the anisotropy is more clear for spheres of larger size. It is observed that the ratio $D_{\|} / D_{\perp}$ varies weakly with the density only for smaller spheres of radius, 0.5 .

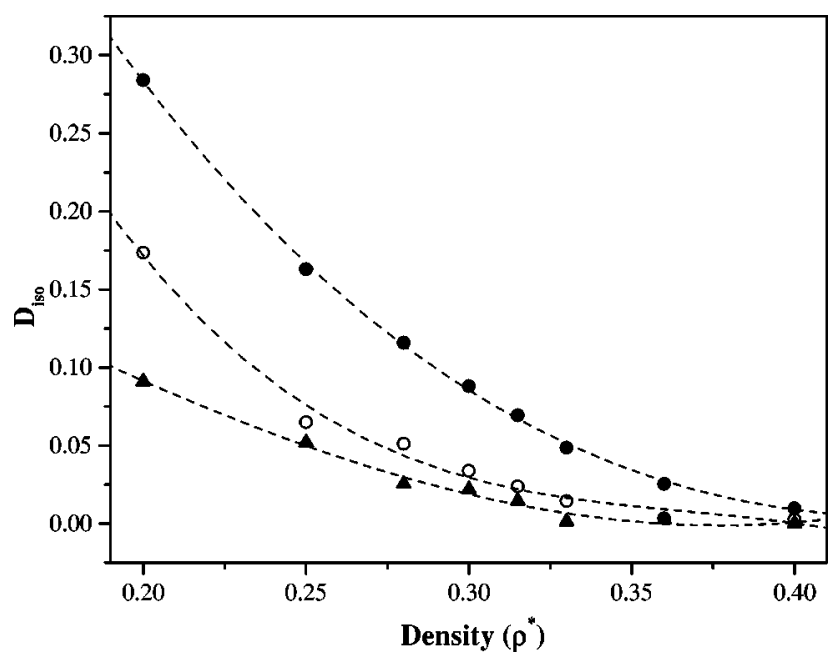

FIG. 4. The total isotropic diffusion coefficient is plotted against the reduced density, $\rho^{*}$ at a reduced temperature $T^{*}=1.0$ for different sizes of spheres. Filled circles: radius $=0.5 \sigma$, open circles: radius $=1.0 \sigma$, and filled triangles: radius $=1.5 \sigma$. The dashed lines are fits to the simulated values.

Figures 3(a), 3(b), and 3(c) show the variation of the parallel and perpendicular components of the diffusion coefficient explicitly for different sizes of the spheres, 0.5, 1.0, and 1.5. Anisotropy is clearly seen to arise very close to the region where the I-N transition occurs.

In Fig. 4, we plot the total diffusion coefficient against density, $\rho^{*}$. This decreases as I-N transition is approached and becomes very small beyond $\rho^{*} \approx 0.33$. Above $\rho^{*}=0.25$, the variation of the diffusion coefficient with density is nearly linear in case of the spheres of radius 1.5 . There is no evidence of any dramatic slowing down of the total diffusion coefficient.

Figure 5 shows the decay of $D_{\perp}$ with density. From a nonlinear curve fit (solid line) of the form

$$
y=y_{0}+A\left|\rho^{*}-\rho_{c}^{*}\right|^{p} .
$$

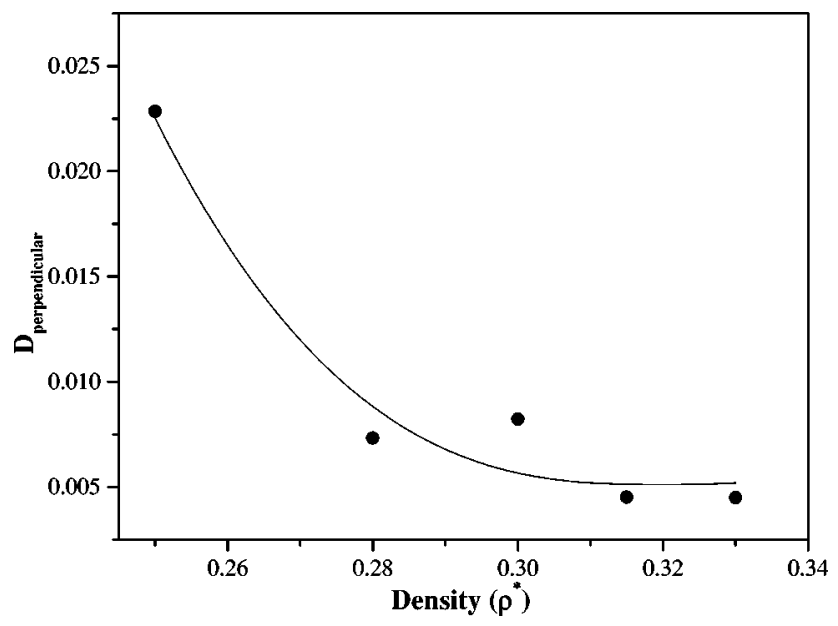

FIG. 5. The plot if $D_{\perp}$ against $\rho^{*}$ at the reduced temperature $T^{*}=1.0$. The filled circles indicate the simulation result and the solid line shows the nonlinear curve fit to the form $y=y_{0}+\left(\rho_{c}^{*}-\rho^{*}\right)^{p}$, with $\rho_{c}^{*}=0.327$ and $p$ $=2.8$. 


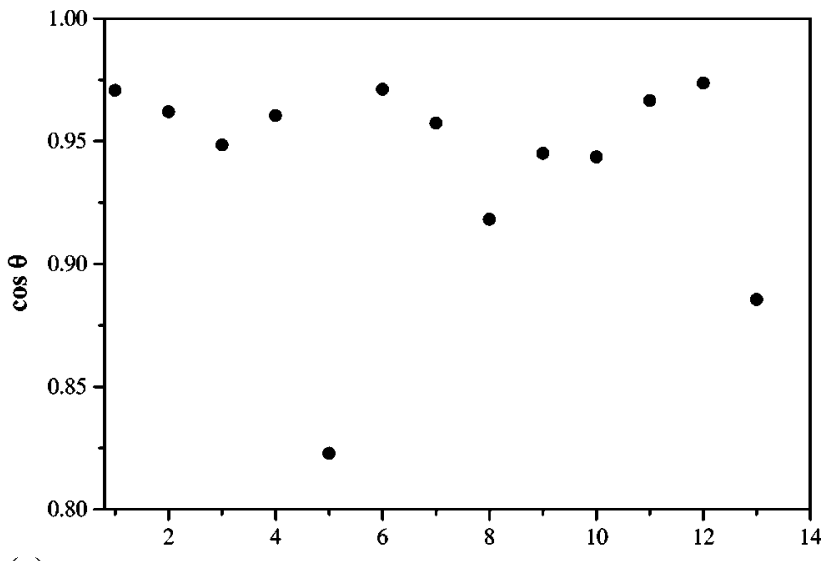

(a)

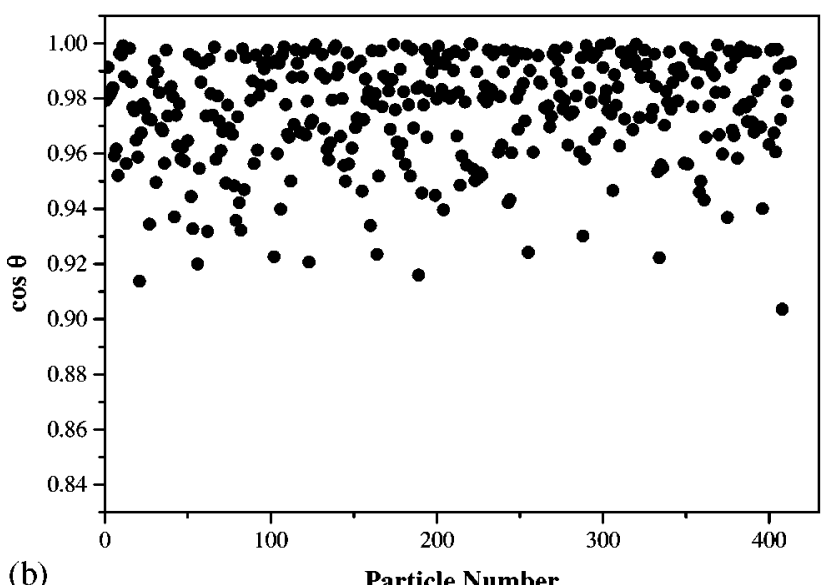

FIG. 6. The cosine of the angle made by the ellipsoid with the nematic director for different ellipsoids at the reduced density, $\rho^{*}=0.4$, temperature $T^{*}=1.0$ and radius of the sphere being 1.0 . (a) shows the variation of cosine of the angle for the ellipsoids at the surface of the sphere while (b) shows that for the ellipsoids which are away from the sphere.

The values of $\rho_{c}^{*}$ and $p$ are calculated which turn out to be 0.327 and 2.8, respectively. While not much significance can be attached to the value of the exponent $p$ (due to neglect of various factors, such as finite size effect) the value of $\rho_{c}^{*}$ is more trustworthy. The mode coupling theory predicts that $D_{\perp}$ varies as below

$$
D_{\perp} \propto\left(\rho_{c}-\rho\right)^{p} .
$$

From the simulation, it is clear that the I-N phase transition takes place at a reduced density, $\rho^{*}=0.315$. However, the nonlinear curve fit to the $D_{\perp}$ versus $\rho^{*}$ shows that the transition occurs at reduced density, $\rho^{*}=0.327$. This is in agreement with the theoretical analyses ${ }^{19,20}$ which find that a weakly first order I-N phase transition intervenes before the divergence of the orientational structure factor, $S_{220}(k)$.

In Figs. 6(a) and 6(b), the orientation of the ellipsoids both at the surface of the sphere as well as away from the sphere with respect to the nematic director has been examined at the reduced density, $\rho^{*}=0.4$, which is in the nematic LC phase. Figure 6(a) shows the cosine of the angle made by the individual ellipsoids [which are at the surface of the sphere within a distance of $1.5 \sigma(\theta)]$ with the nematic director. Figure 6(b) shows the angle made by the remaining ellipsoids with the nematic director which are away from the sphere. From Fig. 6(a), it is clear that orientation of two particles gets disturbed noticably but not significantly and on an average, the nematic ordering is retained. In Fig. 6(b), we see that the orientation of the ellipsoids with respect to the director does not change much. It is observed that on addition of spheres in the system, the LC order gets perturbed only locally at the surface of the sphere.

The order parameter remains very close to zero at very small densities indicating the isotropy in the system and the orientation of the molecules in the system is random. The nematic domains start appearing very close to the I-N transition region which leads to the anisotropy in the system. The anisotropy of the diffusion coefficients of the ellipsoids near the I-N transition or in nematic phase is well known., ${ }^{40,11}$ Here we observe the anisotropy in the diffusion coefficient of the sphere which is clearly seen in Figs. 2, 3(a), 3(b), and 3 (c) and this is strongly dependent on the size of the sphere. The decoupling of the parallel and the perpendicular diffusion coefficients can be clearly seen in Figs. 3(a), 3(b) and 3 (c) near the I-N transition. It is seen that this coupling of the parallel and perpendicular components of the diffusion coefficient depend on the size of the sphere because it occurs at different densities for the spheres with different sizes. The strong size dependence of diffusion shown in Figs. 3 and 4 are in tentative agreement with recent experimental results. ${ }^{15}$

\section{CONCLUSION}

While anisotropic diffusion of tagged ellipsoids in the nematic liquid crystal phase is well-known and has been studied earlier in simulations, ${ }^{4,5}$ the same for diffusion of tagged spheres has not been investigated, to the best of our knowledge. This is not only of theoretical interest, but has been studied recently in experiments as well. ${ }^{15}$

The simulations have revealed two results which are of considerable interest. First, the diffusion becomes anisotropic very close to the I-N transition. This is because it is only very near the transition that the order parameter has a significant nonzero value. Second, the anisotropy in particular and diffusion in general show a very strong sphere size dependence. This can be seen most clearly in Figs. 2 and 3 where the smallest size solute (radius $=0.5$ ) shows a significant anisotropy near the transition, but the least anisotropy in the nematic phase. The largest size solute (radius=1.5) shows different behavior. Here the solute shows detectable anisotropic diffusion before the transition (at the reduced density 0.30) which becomes larger in the nematic phase (Fig. 2). Another result of some importance is the rate of approach of the diffusion to zero which seems to follow a power law behavior. While the fitting performed here should not be taken too seriously, the value of the density where the diffusion goes to zero is found to be 0.327 which appears to agree with the theoretical result ${ }^{20}$ that the system would have faced a criticality if the weakly first order phase transition did not intervene. This criticality is the divergence of the orientational correlation structure factor, $S_{220}(k)$. The analysis of the orientation of the ellipsoids near the surface of the sphere shows that the orientation of some of the ellipsoids get perturbed but still on an average, the LC order is maintained. 
We have presented a mode coupling theory analysis which demonstrates that the translational diffusion of spheres is correlated with the collective rotational dynamics of the nematogens. Analysis also shows that if there is any singular behavior in the density or temperature dependence of the translational diffusion of tagged spheres, then it must come from the rotational diffusion. We have presented a mode coupling theory analysis of the latter which shows that there could be a weak singularity superimposed on the normal density dependence. This seems to be borne out by the nonlinear fitting discussed above. The evidence is not conclusive and needs to be studied in much greater detail.

\section{ACKNOWLEDGMENTS}

The authors thank G. Srinivas, Dr. Sarika Bhattacharyya, and Arnab Mukherjee for much help and discussions. It is a pleasure to thank Professor Michael Fayer and Steve Gottke for many discussions regarding the dynamics in the isotropic phase near the I-N transition. This work was supported in parts by the Department of Science and Technology, and the Council of Scientific and Industrial Research, India.

${ }^{1}$ B. J. Berne and R. Pecora, Dynamic Light Scattering (Wiley, New York, 1976), Chap. 7.

${ }^{2}$ P. G. de Gennes, The Physics of Liquid Crystals (Clarendon, Oxford, 1974).

${ }^{3}$ S. Chandrashekar, Liquid Crystals, 2nd ed. (Cambridge, New York, 1992).

${ }^{4}$ J. Crain and A. V. Komolkin, Adv. Chem. Phys. 109, 39 (1999).
${ }^{5}$ R. Eppenga and D. Frenkel, Mol. Phys. 52, 1303 (1984).

${ }^{6}$ J. G. Gay and B. J. Berne, J. Chem. Phys. 74, 3316 (1981).

${ }^{7}$ E. de Miguel, L. F. Rull, M. K. Chalam, K. E. Gubbins, and F. Van Swol, Mol. Phys. 72, 593 (1991).

${ }^{8}$ S. Ravichandran, A. Perera, M. Moreau, and B. Bagchi, J. Chem. Phys. 109, 7349 (1998).

${ }^{9}$ S. Ravichandran, A. Perera, M. Moreau, and B. Bagchi, J. Chem. Phys. 107, 8469 (1997).

${ }^{10}$ S. Ravichandran and B. Bagchi, J. Chem. Phys. 111, 7505 (1999).

${ }^{11}$ R. Vasanthi, S. Ravichandran, and B. Bagchi, J. Chem. Phys. 114, 7989 (2001).

${ }^{12}$ A. Sengupta and M. D. Fayer, J. Chem. Phys. 102, 4193 (1995).

${ }^{13}$ G. Hinze, D. D. Brace, S. D. Gottke, and M. D. Fayer, J. Chem. Phys. 113, 3723 (2000); J. J. Stankus, R. Torre, and M. D. Fayer, J. Phys. Chem. 97, 9478 (1993).

${ }^{14}$ S. D. Gottke, D. D. Brace, Hu Cang, B. Bagchi, and M. D. Fayer (preprint).

${ }^{15}$ S. G. J. M. Kluijtmans, G. H. Koenderink, and A. P. Philpse, Phys. Rev. E 61, 626 (2000).

${ }^{16}$ U. Balucani and M. Zoppi, Dynamics of Liquid State (Clarendon, Oxford, 1994).

${ }^{17}$ B. Bagchi and S. Bhattacharyya, Adv. Chem. Phys. 116, 67 (2001).

${ }^{18}$ B. Bagchi and A. Chandra, Adv. Chem. Phys. 80, 1 (1991).

${ }^{19}$ A. Perera, P. G. Kusalik, and G. N. Patey, J. Chem. Phys. 87, 1295 (1987); Mol. Phys. 60, 77 (1987); A. Perera, G. Patey, and J. J. Weis, J. Chem. Phys. 89, 6941 (1988).

${ }^{20}$ R. Zwanzig, J. Chem. Phys. 39, 1714 (1963).

${ }^{21}$ The potential has been developed and tested in collaboration with Dr. A. Perera of LPTL, Univ. P. et M. Curie, Jussieu, Paris 75252 (private communication). This potential was used in Refs. 7-10 and shown to give consistent results.

${ }^{22}$ M. P. Allen and D. J. Tildesley, Computer Simulation of Liquids (Clarendon, Oxford, 1987).

${ }^{23}$ J. Viellard-Baron, Mol. Phys. 28, 809 (1974). 